\title{
On the white dwarf distances to galactic globular clusters
}

\author{
M. Salaris ${ }^{1,2}$, S. Cassisi ${ }^{3,2}$, E. García-Berro ${ }^{4,6}$, J. Isern $^{5,6}$, and S. Torres ${ }^{4}$ \\ 1 Astrophysics Research Institute, Liverpool John Moores University, Twelve Quays House, Egerton Wharf, \\ Birkenhead CH41 1LD, UK \\ 2 Max-Planck-Institut für Astrophysik, Karl-Schwarzschild-Straße 1, 85741 Garching, Germany \\ 3 Osservatorio Astronomico di Collurania, via M. Maggini, 64100 Teramo, Italy \\ 4 Departament de Física Aplicada, Universitat Politècnica de Catalunya, c/Jordi Girona Salgado s/n, Módul B-4, \\ Campus Nord, 08034 Barcelona, Spain \\ 5 Institut de Ciències de l'Espai (CSIC), Edifici Nexus, Gran Capitá 2-4, 08034 Barcelona, Spain \\ ${ }^{6}$ Institut d'Estudis Espacials de Catalunya
}

Received 11 January 2001 / Accepted 9 March 2001

\begin{abstract}
We analyze in detail various possible sources of systematic errors on the distances of globular clusters derived by fitting a local template DA white dwarf sequence to the cluster counterpart (the so-called WD-fitting technique). We find that the unknown thickness of the hydrogen layer of white dwarfs in clusters plays a non negligible role. For reasonable assumptions - supported by the few sparse available observational constraints about the unknown mass and thickness of the hydrogen layer for the cluster white dwarfs, a realistic estimate of the systematic error on the distance is within \pm 0.10 mag. However, particular combinations of white dwarf masses and envelope thicknesses - which at present cannot be excluded a priori - could produce larger errors. Contamination of the cluster DA sequence by non-DA white dwarfs introduces a very small systematic error of about $-0.03 \mathrm{mag}$ in the $M_{V} /(V-I)$ plane, but in the $M_{V} /(B-V)$ plane the systematic error amounts to $\sim+0.20$ mag. Contamination by white dwarfs with helium cores should not influence appreciably the WD-fitting distances. Finally, we obtain a derivative $\Delta(m-M)_{V} / \Delta E(B-V) \sim-5.5$ for the WD-fitting distances, which is very similar to the dependence found when using the Main Sequence fitting technique.
\end{abstract}

Key words. distance scale - globular clusters: general - stars: white dwarfs

\section{Introduction}

Globular cluster stars are possibly the most suitable objects to be used in order to constrain the age of the universe. Since stars in a given globular cluster are coeval and share the same initial chemical composition, the turnoff brightness derived from the globular cluster colourmagnitude diagram (CMD) provides straightforwardly the cluster age: if the cluster distance is known, one only has to compare the observed cluster turn-off brightness with the correspondent quantity predicted by theoretical isochrones with the appropriate chemical composition in order to derive a firm estimate of its age.

The globular cluster distance scale is however still the subject of an intense debate. Main Sequence (MS) fitting distances using metal poor Hipparcos subdwarfs with well determined parallaxes provide long distances implying globular cluster ages of the order of 12 Gyr (see, e.g., Gratton et al. 1997). The same long distances are derived

Send offprint requests to: M. Salaris,

e-mail: ms@astro.livjm.ac.uk by Salaris \& Weiss (1997, 1998), Mazzitelli et al. (1995), Cassisi et al. (1999) when using as standard candles theoretical Zero Age Horizontal Branch (ZAHB) models, while shorter distances are obtained from the ZAHB models of Vandenberg et al. (2000). On the other hand, the calibration of the RR Lyrae stars absolute brightness using the statistical parallax methods (see, for instance, Fernley et al. 1998 and Luri et al. 1998) provides consistently much shorter distances and therefore higher globular cluster ages. Taking these results at face value, the uncertainty of the globular cluster distance scale appears to be still of the order of $0.25-0.30 \mathrm{mag}$, which translates into an indetermination on the globular cluster age of about $20 \%$ - see the discussion in Renzini et al. (1996).

An alternative empirical method to derive globular cluster distances has been recently applied to the galactic globular cluster NGC 6752 (Renzini et al. 1996). The distance indicator is in this case a template local sequence of white dwarf stars with effective temperature $\left(T_{\text {eff }}\right)$ ranging between 10000 and $20000 \mathrm{~K}$, and precise parallax measurements, which is fitted to the dereddened cluster white 
dwarf sequence. The vertical shift applied to the local template sequence in order to fit the sequence of the cluster provides its distance modulus. We shall refer to this technique as the WD-fitting technique, because of its close analogy with the MS-fitting technique.

A key assumption of this method is that the white dwarfs of the local template sequence - which, in the case of the paper by Renzini et al. (1996), have an estimated average mass $M=0.515 M_{\odot}$ - are totally equivalent to the white dwarfs of the cluster. If this is the case, the main advantage of the WD-fitting technique over the MS-fitting one is that it is in principle independent of the knowledge of the initial chemical composition of the globular cluster, since all white dwarfs have virtually metal free atmospheres, and therefore it avoids the uncertainties introduced in the MS-fitting method by the colour corrections that have to be necessarily applied to the local subdwarfs in order to precisely match the metallicity of the globular cluster under scrutiny.

Another potential advantage of the WD-fitting method with respect to the MS-fitting technique is, according to Renzini et al. (1996), that local white dwarfs are more abundant than metal poor subdwarfs, and therefore it is possible in principle to have a larger sample of local calibrators. However, since white dwarfs are much dimmer than the subdwarfs used in the MS-fitting technique, up to now it is possible to apply the WD-fitting technique only through HST observations of relatively close globular clusters.

Finally it should be mentioned as well that in the $T_{\text {eff }}$ range considered by Renzini et al. (1996), local white dwarfs appear in two types, either as the so-called DA spectral type, which are characterized by an envelope made up of pure $\mathrm{H}$ (on top of a He layer), or as the non-DA type, which is characterized by an almost pure He envelope and possibly, at least for $20 \%$ of them, traces of $\mathrm{H}$ with abundances of the order of $10^{-4}$ by number, or even less - see the review by Koester \& Chanmugam (1990), and references therein. For local white dwarfs the number ratio of DA versus non-DA is of the order of 4:1 in this temperature range. Thus, the heterogeneity of the local sample of white dwarfs could potentially have undesired effects on the determinations of the ages and distances of globular clusters using the WD-fitting method.

Because of the potential advantages offered by the WD-fitting method, we analyze in detail the possible systematic uncertainties involved in this technique due to the poor knowledge of parameters affecting the CMD location of the template and globular cluster white dwarfs, expanding upon the discussion by Renzini et al. (1996). We pay particular attention to the role played by differences in the thickness of the surface $\mathrm{H}$ and He layers on the final distance determination - which has not yet been explored -, to the possible differences between white dwarfs in clusters with predominantly blue or red Horizontal Branches (HB), and also to the differences obtained when using DA or non-DA model atmospheres, or evolutionary models of white dwarfs with He cores. All along our analysis we use evolutionary white dwarf models in a purely differential way.

The paper is organized as follows. In Sect. 2 we briefly describe the theoretical models, while in Sect. 3 the influence of the various parameters affecting the CMD of white dwarfs is assessed. An exhaustive estimate of the systematic errors involved in the WD-fitting technique is derived in Sect. 4, which is followed by Sect. 5 were we draw our conclusions.

\section{The models}

The stellar evolution computations have been performed using the same code and the same input physics described in Salaris et al. (2000). We just recall here that in the temperature range we are dealing with $\left(T_{\text {eff }}\right.$ well above $6000 \mathrm{~K}$ ) the OPAL radiative opacities (Iglesias \& Rogers 1993) with $Z=0$ are used, together with the conductive opacities by Itoh et al. (1983) supplemented by the Hubbard \& Lampe (1969) ones; the boundary conditions for the integration of the stellar structure have been derived in this case from a grey $T(\tau)$ relationship, which is completely adequate at these temperatures - see, for instance, Hansen (1999) and Salaris et al. (2000). The equation of state for the $\mathrm{H}$ and $\mathrm{He}$ envelopes is that of Saumon et al. (1995), while the equation of state for the carbon-oxygen (CO) core is from Segretain et al. (1994); neutrino energy losses have been taken from Itoh et al. (1996). Bolometric luminosities and effective temperatures have been transformed into $V$ magnitudes and colours using the relationships by Bergeron et al. (1995).

We have computed white dwarf model sequences for several masses, CO stratifications - unless stated otherwise our reference CO stratification is that from Salaris et al. (1997) - and various $\mathrm{H}$ and/or He envelope thicknesses, which will be described in the following section. For all the computations an initial model with the selected CO profile and envelope structure was converged at $\log \left(L / L_{\odot}\right) \sim 2.0$ and evolved down to sufficiently low temperatures.

\section{The white dwarf sequence location in the CMD}

As already mentioned in Sect. 1, the WD-fitting method is based on the fitting of a template white dwarf sequence to the correspondent globular cluster one. The key ingredient to derive reliable distances (and hence ages) is to ensure that the local white dwarfs included in the template sequence are homogeneous with the globular cluster ones.

All stars in a globular cluster are coeval; therefore, the luminosity (and, thus, $T_{\text {eff }}$ ) of a white dwarf of a given mass is constrained by the fact that the sum of its cooling age $\left(t_{\text {cool }}\right)$ plus the evolutionary time of its progenitor $\left(t_{\text {prog }}\right)-$ which, to a good approximation, is equal to its main sequence lifetime, since subsequent evolutionary phases are much shorter - must be equal to the globular cluster age $\left(t_{\mathrm{GC}}\right)$. It is immediately clear that one also has 


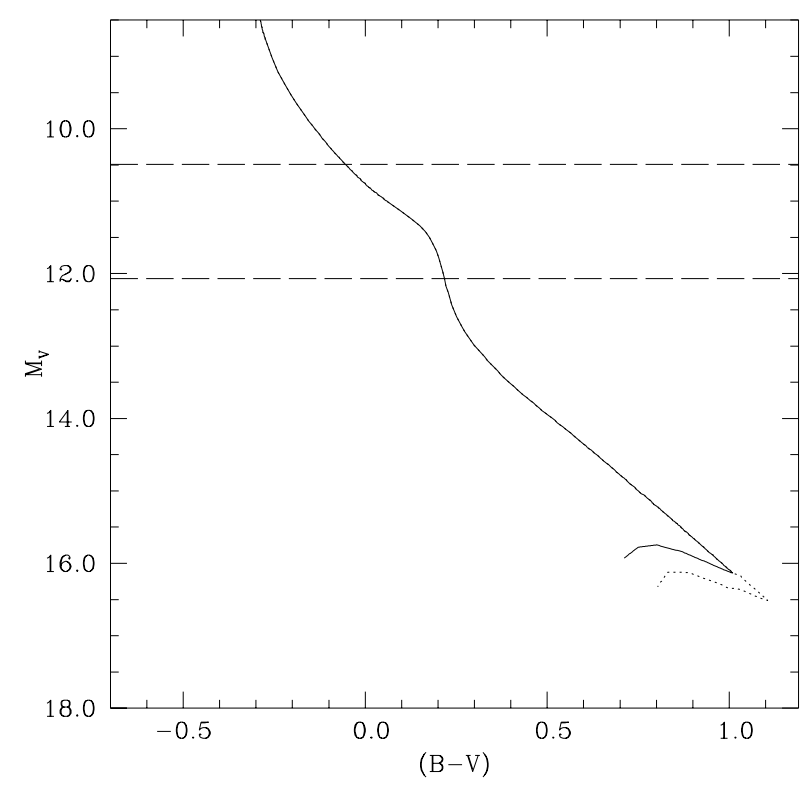

Fig. 1. Colour magnitude diagram of two cluster white dwarf cooling sequences with ages $t=10$ (solid line) and 12 Gyr (dotted line), and solar metallicity, for the white dwarf progenitors from Salaris et al. (2000). The horizontal dashed lines mark the region with $T_{\text {eff }}$ between 20000 and $10000 \mathrm{~K}$

to assume an initial-final mass relationship, that is, a relation between the white dwarf mass and the initial mass of its progenitor. In Fig. 1 we show, as an example, the location of the white dwarfs of an old cluster of initial solar metallicity and ages of 10 and 12 Gyr, respectively, in the $M_{V} /(B-V)$ plane, as predicted by the theoretical models (Salaris et al. 2000). The horizontal lines mark the region where white dwarfs have effective temperatures between 10000 and $20000 \mathrm{~K}$, which is the temperature range of the template field white dwarfs used by Renzini et al. (1996) to derive the distance to NGC 6752.

The initial-final mass relationship has been derived considering the CO core masses after the first thermal pulse from the evolutionary models of Salaris et al. (1997). This initial-final mass relationship provides white dwarf masses nearly constant and equal to $0.54 M_{\odot}$ for progenitor masses up to $\sim 2.5 M_{\odot}$, and then increasing up to $1.0 M_{\odot}$ when the progenitor mass reaches $7.0 M_{\odot}$. It can be easily seen in Fig. 1 that the cluster white dwarf sequences show a pronounced turn to the blue at their dimmer end which, as the age of the cluster increases, is located at increasingly larger magnitudes. Until this blueturn the white dwarf sequence is almost coincident with the cooling track of the $\sim 0.54 M_{\odot}$, while the blue-turn is due to the contribution of more massive white dwarfs. It is easy to understand this behaviour by recalling that at each brightness along the cooling sequence the constraint $t_{\mathrm{GC}}=t_{\mathrm{cool}}+t_{\mathrm{prog}}$ has to be valid. Since $t_{\text {cool }}$ is very short at the bright end of the cooling sequence, and practically negligible with respect to $t_{\mathrm{GC}}, t_{\mathrm{prog}}$ and, hence, the progenitor mass has to be, to a very good approximation, constant for a large magnitude range, and very close to the turn-off mass. On the contrary, towards the dim end

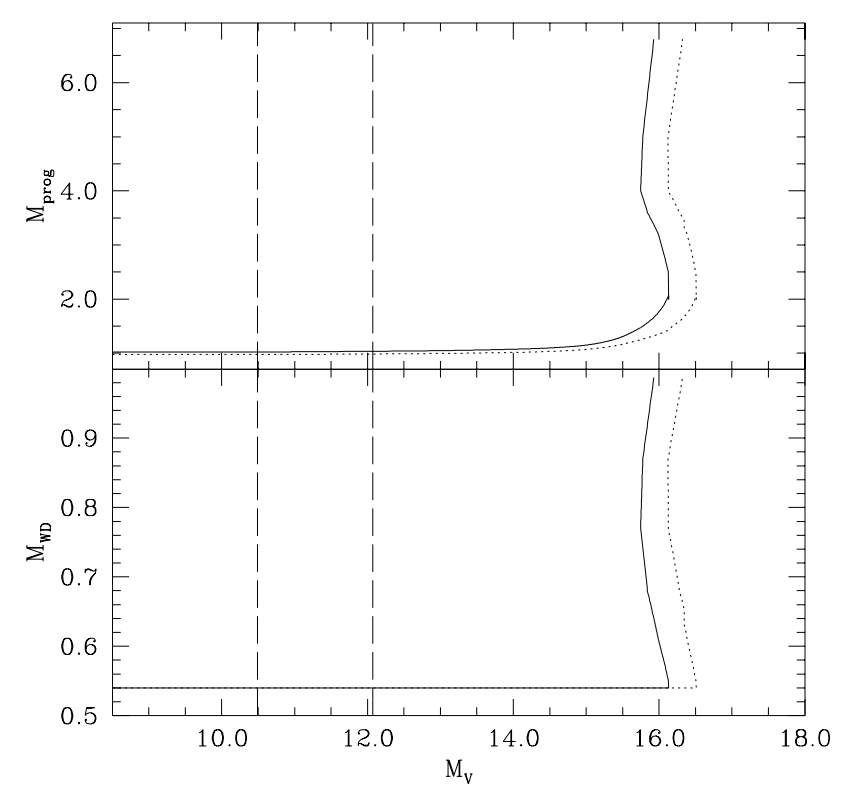

Fig. 2. Progenitor mass (upper panel) and white dwarf mass (lower panel) as a function of $M_{V}$ for the cluster cooling sequences displayed in Fig. 1. As in the previous figure the vertical dashed lines mark the region with $T_{\text {eff }}$ between 20000 and $10000 \mathrm{~K}$

of the cooling sequence $t_{\text {cool }}$ becomes a sizeable fraction of $t_{\mathrm{GC}}$ and, thus, the contribution of the white dwarfs coming from higher mass progenitors (and, consequently, with smaller $t_{\text {prog }}$ ) is quite apparent. A similar feature is expected for the halo white dwarf population (Isern et al. 1998).

This feature can also be seen in Fig. 2 (upper panel) where we show the run of the initial masses as a function of the $M_{V}$ magnitude along the cluster white dwarf sequences shown in Fig. 1 . In the $T_{\text {eff }}$ range we are dealing with (marked, as in Fig. 1, by vertical dashed lines), the initial mass is almost constant - as well as, due to our selected initial-final mass relationship, the actual white dwarf mass shown in the lower panel. It is remarkable that even two magnitudes below the lower limit of this effective temperature range the initial mass of white dwarf progenitors is still constant. This result (the constancy of the progenitor mass of white dwarfs contributing to the effective temperature range, not its actual value) is completely general, that is, independent of the adopted initialfinal mass relationship, since for all possible white dwarf masses the evolutionary times are very fast at these high temperatures; moreover, this result is also independent of the initial metallicity of the white dwarf progenitors and therefore valid also in the case of white dwarf progenitors with lower metallicities, typical of galactic globular clusters.

Since the progenitor mass is constant, one can then reasonably expect that, even in the case of a completely different initial-final mass relationship, the mean white dwarf mass and the spread around this value is constant, and equal to the values attained at the beginning of the cooling sequence. This behaviour is very different from 
what happens in field disk white dwarfs. In this case, because of the ongoing star formation processes, very different white dwarf masses can populate the same $T_{\text {eff }}$ range, depending on the age of their progenitors.

There are in principle four quantities which can introduce a systematic error in the white dwarf distance determination to globular clusters, provided that there are significant differences between the properties of the local template white dwarf sequence and the globular cluster one, namely:

i) The mass of the white dwarf cooling sequence;

ii) The chemical composition of the envelope (DA, nonDA);

iii) The thickness (in mass) of the envelope;

iv) The chemical stratification of the core (different CO profiles or, even, He--core white dwarfs).

In the following we are going to discuss the influence of these four parameters on the CMD location of white dwarf cooling sequences in the $M_{V} /(B-V)$ (hereinafter $B V$ ) and $M_{V} /(V-I)$ (hereinafter $V I$ ) planes, which are actually the most popular CMDs employed for studying cluster white dwarfs - see, e.g., Renzini et al. (1996); Richer et al. (1997); and Von Hippel et al. (2000). We consider as a reference the $T_{\text {eff }}$ range between 10000 and $20000 \mathrm{~K}$, already selected by Renzini et al. (1996) for their distance determination. In this temperature range the white dwarf sequence is sufficiently populated and bright enough to be detected with a reasonable photometric error in close globular clusters, using the HST or giant ground based telescopes.

\subsection{The mass of the white dwarf sequence}

Stars in a globular cluster lose their mass along the Red Giant Branch (RGB) due to stellar winds. The amount of mass lost during this phase is not the same for each red giant star, and this, in turn, is the origin of the extended horizontal branches (HB) observed in Galactic globular clusters; this also means that the mass evolving along the RGB (which at the beginning of the red giant phase is almost equal to the mass of the star at the cluster turnoff) can give birth to HB stars with different values of the total mass, but with the same initial He core mass. These different HB stars will all end up as white dwarfs with a mass range which is constrained mainly by the size of the helium core at the beginning of the He-burning phase and by the mass loss processes along the subsequent Asymptotic Giant Branch (AGB) phase.

Renzini et al. (1996) summarize the results about the few semiempirical determinations of white dwarf masses in galactic globular clusters, providing a very narrow range of values, namely $0.53 \pm 0.02 M_{\odot}$. This range of values nicely overlaps with current determinations of the $\mathrm{CO}$ core mass at the first thermal pulse derived from stellar evolutionary models - see, e.g., Wagenhuber (1996) and Salaris et al. (1997) - for initial masses of about $0.8-1.0 M_{\odot}$. Moreover, it is also in agreement with determinations of the mass distribution of nearby field white dwarfs, which peaks at about $0.55 M_{\odot}$ (Bragaglia et al. 1995; Reid 1996). However, regarding this last result, the reader should keep also in mind the recent results of Bergeron et al. (2000), who find a mean mass of field white dwarfs about $0.10 M_{\odot}$ higher at lower $T_{\text {eff }}$ values.

Very recently however, Alves et al. (2000) derived semiempirically the mass of the central star in a planetary nebula of the globular cluster M 15, obtaining a value $0.60 \pm 0.02 M_{\odot}$. It is not clear yet if this result is an indication of a globular cluster initial-final mass relationship with a larger dispersion than that derived by Renzini et al. (1996), or it is due (as discussed by the authors) to an increment of the mass through an interaction in a close binary system. We just recall here that there is also another possible indication of a large dispersion for the initial-final mass relationship in the results obtained by Reid (1996) when considering the white dwarf population of the Hyades open cluster.

Another important piece of evidence must also be taken into account. The determination of the mass range $0.53 \pm 0.02 M_{\odot}-$ and the results of Alves et al. (2000), as well - is mainly based on the maximum brightness of AGB stars and on the luminosity of post-AGB stars, which can be directly related to the mass of the degenerate $\mathrm{CO}$ core prior to the beginning of the cooling phase. However, there are several globular clusters with an horizontal branch morphology showing the presence of an extended blue tail, as in the case of NGC 6752. Regardless of the physical mechanism(s) producing the stars which populate the hot side of the HB in globular clusters, it is well known that they are stars which have lost a large amount of their envelope during the RGB phase. At the end of the He-burning phase these stars, depending on the mass of their residual $\mathrm{H}$-rich envelope, can behave as post-Early AGB structures or as AGB-manqué ones - see, for instance, Greggio \& Renzini (1990) - and do not experience the thermally pulsing phase on the AGB as most massive HB stars do. On theoretical grounds, the minimum (initial) HB mass which does not experience the AGB thermal pulses is a quite robust prediction (Dorman et al. 1993; Bono et al. 1997), being equal to $\approx 0.52 M_{\odot}$. Since the mass of the CO core at the end of the HB phase is of the order of $0.45 M_{\odot}$, globular clusters with extended blue tails can produce also white dwarfs with masses in the range $0.45-0.52 M_{\odot}$, on average $\approx 0.05 M_{\odot}$ less massive than the white dwarf progeny of stars climbing up the AGB. Thus, it is worth considering the possibility of different masses for the globular cluster cooling sequences.

Turning now our attention to the CMD location of white dwarfs of different masses, it is well known from relatively simple physical considerations that the larger the white dwarf mass is, the smaller its radius is. In Fig. 3 we display in the $B V$ and $V I$ planes, for effective temperatures between 10000 and $20000 \mathrm{~K}$, two representative white dwarf cooling tracks with masses equal to 0.54 and $0.61 M_{\odot}$. The higher mass is shifted to higher $M_{V}$ values. 


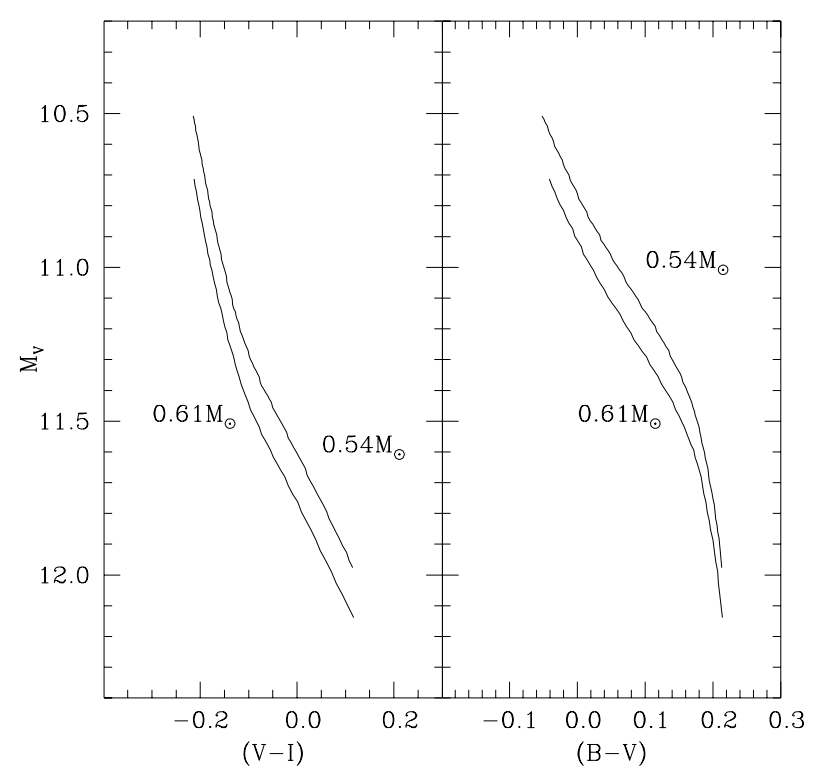

Fig. 3. White dwarf cooling tracks in the $B V$ and $V I$ planes for masses equal to $0.54 M_{\odot}$ and $0.61 M_{\odot}$, and $T_{\text {eff }}$ between 20000 and $10000 \mathrm{~K}$

The derivative $\Delta M_{V} / \Delta\left(M / M_{\odot}\right)$ equals to 2.3 for masses between 0.45 and $0.60 M_{\odot}$.

\subsection{The chemical composition of the envelope}

As already mentioned in the introduction, field white dwarfs with $T_{\text {eff }}$ between $\sim 20000$ and $10000 \mathrm{~K}$ can be present either in the DA or non-DA types, with a number ratio of DA to non-DA equal to 4. Our selected standard envelope thicknesses (see next subsection) are $\log q(\mathrm{H})=-4.0$ and $\log q(\mathrm{He})=-2.0$ for DA white dwarfs, and $\log q(\mathrm{He})=-3.5$ for the non-DA spectral type, where $q(\mathrm{H})$ and $q(\mathrm{He})$ indicate the ratio of the mass contained in the $\mathrm{H}$ and $\mathrm{He}$ envelope layers to the total white dwarf mass, respectively.

The envelope chemical composition strongly affects not only the white dwarf cooling times, but also their location in the CMD, and the precise shape of the cooling track, as clearly shown in Fig. 4 where we show the cooling tracks for a $0.54 M_{\odot}$ DA white dwarf (solid line) and a non-DA white dwarf of the same mass. It is evident that in the $B V$ plane DA white dwarfs are brighter at a fixed colour. This is clearly confirmed by observations of local DA white dwarfs, see e.g. panel 2 of Fig. 1 in Renzini et al. (1996), while the reverse happens in the VI plane. Moreover, the separation between DA and non-DA white dwarfs is larger in the $B V$ plane, making possibly more evident their identification in a given globular cluster. $M_{V}$ differences of up to $\sim 1 \mathrm{mag}$ at a fixed $B V$ value are possible in the $B V$ plane, while they are a factor $\sim 5$ smaller in the $V I$ plane. Also the shape of the DA and non-DA cooling sequences is different, especially in the $B V$ plane.

So far, we have considered DA and non-DA white dwarfs having their very outer layers made of either pure hydrogen or pure helium. However, observations suggest

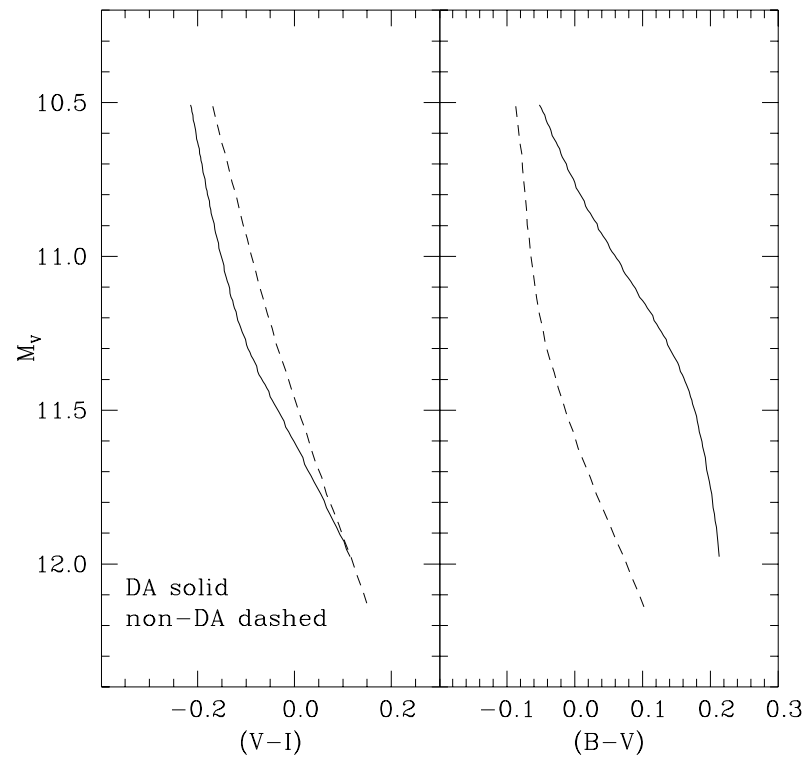

Fig. 4. Same as Fig. 3 but for a white dwarf of $0.54 M_{\odot}$ either DA or non-DA (see text for details)

that in this temperature range DA white dwarfs can have a small amount of helium in their spectra, of the order of $10^{-5}$ by number, and that $20 \%$ of non-DA white dwarfs show some hydrogen in their envelopes, with abundances of the order of $10^{-4}$ by number at most. We have tested the effect of these small admixtures of helium in the outer envelope of DA white dwarfs, and of hydrogen in the envelope of non-DA white dwarfs, by computing appropriate models for a $0.61 M_{\odot}$ white dwarf, and using the results of Bergeron et al. (1995) about the influence of $\mathrm{H}-\mathrm{He}$ mixtures on the derived white dwarf colours. We have found negligible variations of the CMD location with respect to the case of our standard He-free DA white dwarf envelopes and $\mathrm{H}$-free non-DA atmospheres.

\subsection{The thickness of the envelope}

The thicknesses of the hydrogen and helium envelopes of white dwarfs has been the subject of many investigations during the last decade. The thickness of these layers is a key ingredient to determine the evolution of the white dwarf since, due to their opacity, they basically regulate the energy loss rate of the isothermal, highly conductive electron degenerate core. Moreover, the envelope thickness also affects the radius of the white dwarf at a given effective temperature. As discussed by D'Antona \& Mazzitelli (1990), due to the unknown details of the mass loss process during the AGB phase and the planetary nebula ejection, theoretical evolutionary models cannot yet provide too stringent predictions about the thickness of the hydrogen and helium layers surrounding the degenerate CO core.

Observational constraints based on spectroscopic (Barstow et al. 1993) as well as on asteroseismological (Clemens 1995) analyses suggest that local field DA white dwarfs have typically hydrogen layers of about 


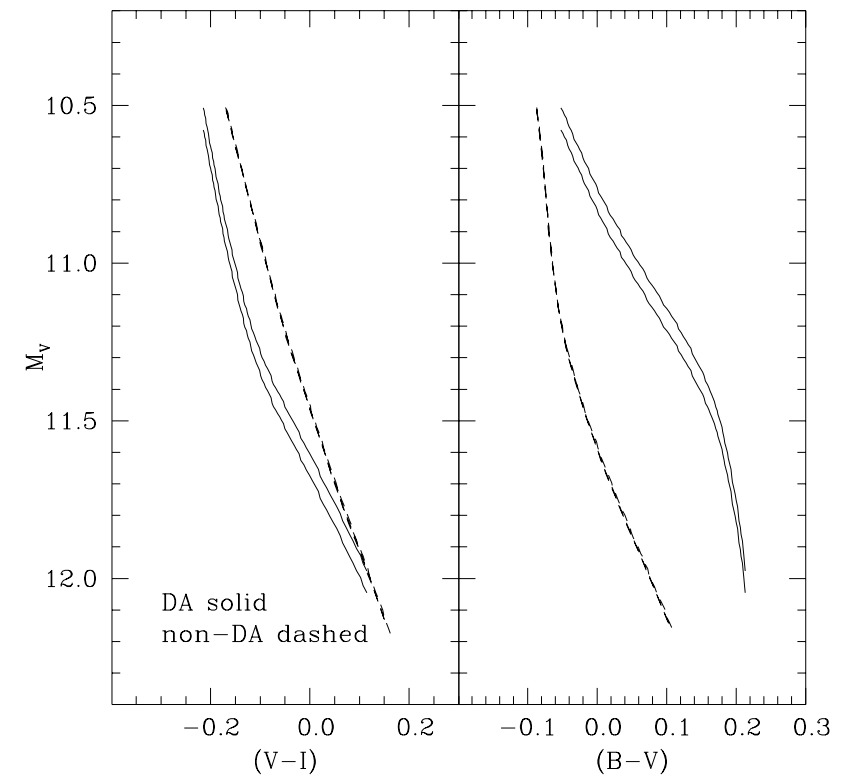

Fig. 5. Same as Fig. 4 but for varying thicknesses of the $H$ and He envelopes (see text for details)

$\log q(\mathrm{H})=-4.0$. On the other hand, the study of the mass-radius relationship for a sample of field white dwarfs with known Hipparcos parallaxes provides indications that the thickness of the hydrogen layers spans a range of values within $\log q(\mathrm{H}) \sim-4.0$ and $\log q(\mathrm{H}) \sim-7.0$ (Provencal et al. 1998). As for the thickness of the helium layer below the hydrogen envelope the assumed reference value comes basically from stellar evolution constraints, and it is $\log q(\mathrm{He}) \sim-2.0$ (Hansen 1999 and references therein). We have verified, by computing white dwarf cooling tracks with $\log q(\mathrm{He})$ decreased by 1 dex (keeping the total white dwarf mass constant) that the location in the CMD of DA white dwarfs is basically unchanged. As for non-DA white dwarfs, estimates of the envelope thickness range between $\sim 10^{-4} M_{\odot}$ and $\sim 10^{-2} M_{\odot}$ (Pelletier et al. 1986; MacDonald et al. 1998).

All these results are however for field white dwarfs; no indications exist yet about $\log q(\mathrm{H})$ and $\log q(\mathrm{He})$ in globular clusters, apart from the fact that theoretical evolutionary models of white dwarfs coming from blue HB progenitors - in the hypothesis that mass loss during the He-burning phases is negligible - predict an upper limit to the thickness of the very outer $\mathrm{H}$ layers ranging from $\log q(\mathrm{H}) \sim-3.5$ to -4.0 (Castellani et al. 1994a).

In Fig. 5 we show the effect of varying the thickness of the external $\mathrm{H}$ and He layers for, respectively, a DA and and a non-DA white dwarf of $0.54 M_{\odot}$. In the case of nonDA white dwarfs our reference value is $\log q(\mathrm{He})=-3.5$, which we have changed by \pm 1.0 dex, obtaining virtually no variation in the location of the cooling track (the three tracks perfectly overlap in Fig. 5). In the case of $\mathrm{H}$ envelopes the situation is different, since the thickness of the $\mathrm{H}$ layers affects appreciably the location of the track. In Fig. 5 representative models with $\log q(\mathrm{H})=-4.0$ and $\log q(\mathrm{H})=-6.0$ are displayed; a reduction of the thickness

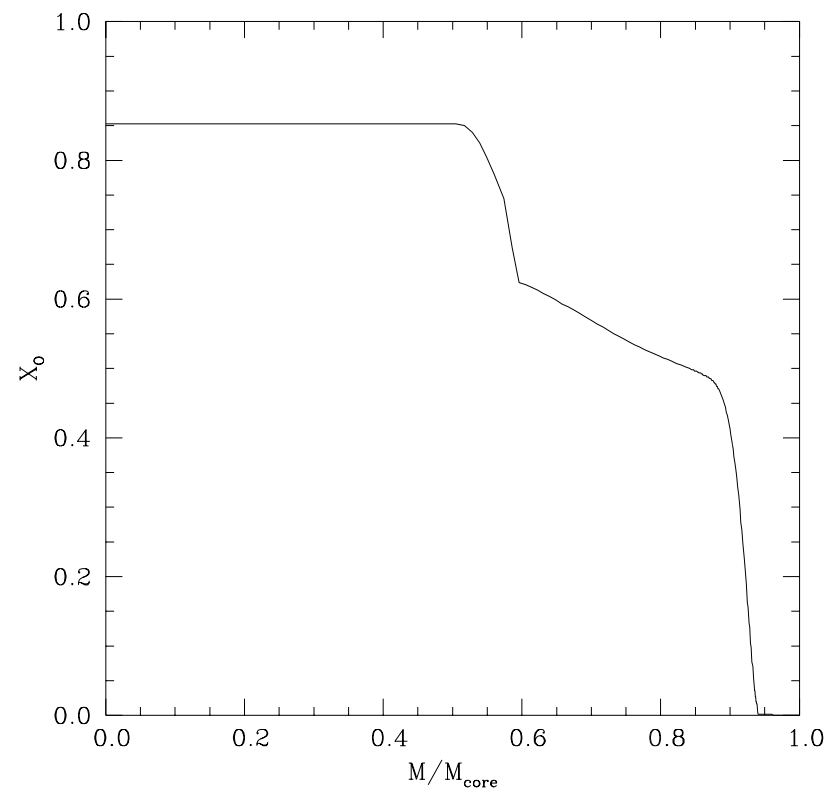

Fig. 6. Profile of the oxygen mass fraction $\left(X_{\mathrm{O}}\right)$ along the CO core of our reference white dwarf model of $0.54 M_{\odot}$. The carbon abundance, $X_{\mathrm{C}}$, is $X_{\mathrm{C}}=1-X_{\mathrm{O}}$

of the $\mathrm{H}$ envelope shifts the track - at a constant value of the colour - towards higher $M_{\mathrm{V}}$ values, with a derivative $\Delta M_{V} / \Delta \log q(\mathrm{H}) \sim-0.035$ for $\log q(\mathrm{H})$ ranging between -4.0 and -7.0 .

\subsection{The chemical stratification of the CO core}

The chemical stratification of the $\mathrm{CO}$ core may potentially affect as well the CMD location of the white dwarf track since the the mass-radius relationship also depends on the electron mean molecular weight of the electrondegenerate core. To this regard, it is important to realize that the value of the $\mathrm{CO}$ ratio along the white dwarf core is subject to some uncertainties due to our poor knowledge of the value of the ${ }^{12} \mathrm{C}(\alpha, \gamma){ }^{16} \mathrm{O}$ reaction rate - see the detailed discussions in Salaris et al. (1997) or Cassisi et al. (2001), and references therein. Moreover, for a given value of the ${ }^{12} \mathrm{C}(\alpha, \gamma){ }^{16} \mathrm{O}$ reaction rate, the $\mathrm{CO}$ profile is possibly a function of the initial metallicity of the white dwarf progenitor (Umeda et al. 1999).

As a numerical test, and in order to mimic these effects, we have computed two cooling tracks for a $0.54 M_{\odot}$ white dwarf, where, for the first case, we have substituted our reference CO stratification shown in Fig. 6 with a flat profile with $50 \%$ of $\mathrm{C}$ and $50 \%$ of $\mathrm{O}$ by mass, whereas in the second case we have inverted the role of $\mathrm{C}$ and $\mathrm{O}$, preserving the shape of the initial chemical profile. The resulting cooling tracks are however negligibly affected by these changes in the effective temperature range we are dealing with. This means that, regardless of the still large uncertainty affecting the ${ }^{12} \mathrm{C}(\alpha, \gamma){ }^{16} \mathrm{O}$ reaction rate, and regardless of the possible effects on the $\mathrm{CO}$ profile of the initial progenitor metallicity, the CMD location of the 
bright portion of the white dwarf cooling sequence is not appreciably affected.

\subsection{He-core white dwarfs}

He-core white dwarfs are the byproduct of strong mass loss along the red giant phase (due for example to the interaction with a binary companion), which strips out the $\mathrm{H}$ envelope on top of the $\mathrm{H}$ burning shell before the degenerate He core reaches the critical mass to experience the He flash and the subsequent quiescent He burning phase (Kippenhahn et al. 1967; Castellani et al. 1994b; Hansen \& Phinney 1998). Their mass can range from a value close to the degenerate He core-mass at the red giant tip (about $0.50 M_{\odot}$ for the most metal poor globular clusters as M 92, decreasing down to about $0.48 M_{\odot}$ for the metal rich ones as 47 Tuc) down to masses of the order of $0.20 M_{\odot}$. There is possibly at least one direct observational indication that He-core white dwarfs contribute to the cooling sequence of globular clusters (Moehler et al. 2000).

In Fig. 7 we show the CMD location of a $0.45 M_{\odot} \mathrm{He}$ core white dwarf with a $\mathrm{H}$ surface layer of $\log q(\mathrm{H})=-4.0$. In the same plot our reference DA and non-DA model sequences of $0.54 M_{\odot}$ are also shown. The He-core white dwarf mass is close to the upper possible value, lower masses being shifted to the red side of the CMD. As expected purely on the base of the mass difference with respect to the DA white dwarf with a $\mathrm{CO}$ core plotted in the figure, the He-core white dwarf is shifted to higher brightnesses at a fixed colour. This feature is reminiscent of the two bright white dwarfs discarded by Renzini et al. (1996) in their fitting procedure - compare the $B V$ panel in Fig. 7 with Fig. 1 of Renzini et al. (1996) - because they were clearly located to the right side of the main DA cluster sequence. In the $V I$ plane the He-core white dwarf sequence overlaps with the non-DA one at the bright end of the $T_{\text {eff }}$ interval. In the $B V$ plane, due to the steeper slope of the $\mathrm{H}-$ envelope cooling sequences, the $\mathrm{He}-$ core white dwarf is on average closer in colour to the $\mathrm{CO}$ one than in the VI plane.

\section{Systematic errors on the WD fitting distances}

In this section we quantify the possible systematic errors on the distances derived from the WD-fitting technique taking into account the results previously discussed. We study the cases in which, due to our lack of precise empirical or theoretical determinations, the parameters which determine the position of the white dwarf sequence on the CMD are possibly different in the local template and in the globular cluster sequence, but within the range allowed by independent observations. Because the ratio between the number of DA and non-DA white dwarfs in the field is $4: 1$ (and presumably this ratio is the same in globular clusters) one is forced to use the cooling sequences of DA white dwarfs in order to build the template sequence. This, in turn, implies that an important role is played by the thickness of the $\mathrm{H}$ surface layers. To this date, we do not have

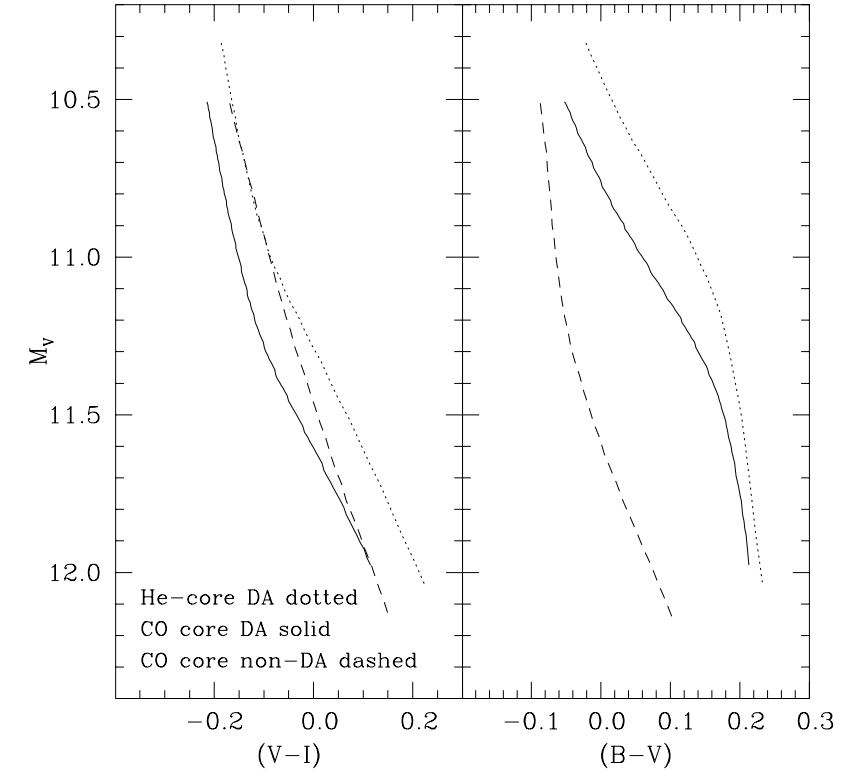

Fig. 7. Comparison of the CMD of a He-core white dwarf, with $\mathrm{H}$ envelope and $M=0.45 M_{\odot}$, with our reference DA and non-DA sequences

any empirical indication about the value of $q(\mathrm{H})$ for white dwarfs in globular clusters, and about its dependence on the HB morphology. In principle, there could be sizeable systematic differences due, for example, to the fact that in globular clusters with blue HB (like M 92 and NGC 6752) the main component of the white dwarf population could be produced by stars not experiencing the AGB phase, in contrast with white dwarfs populating red $\mathrm{HB}$ globular clusters (like 47 Tuc). We are forced, therefore, to treat the thickness of the envelope layers as a free parameter, in the hypothesis that the possible range of values is within the range spanned by field white dwarfs.

\subsection{Uncertainties on the template sequence}

Since the mass range for white dwarfs in globular clusters appears to be mainly in the range $0.53 \pm 0.02 M_{\odot}$, it is clear that the template white dwarf sequence should be made by stars within this mass range. However, there are almost no field white dwarfs suitable for the WD-fitting method, for which the value of the mass is derived empirically (like, for instance, the case of white dwarfs in close binary systems, as Sirius B). In order to build the template DA white dwarf sequence, one needs therefore to employ mass determinations obtained from semiempirical methods, as discussed in Bragaglia et al. (1995). It is possible to determine spectroscopical values for the gravity $(g)$ and $T_{\text {eff }}$ of a sample of local white dwarfs, and then derive the masses employing theoretical $\log g-T_{\text {eff }}$ relationships. However, the assumed thicknesses of the envelope layers of

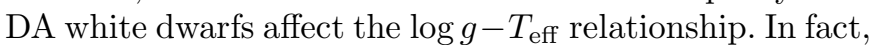
it can be shown that a uniform distribution of $\log q(\mathrm{H})$ between -4.0 and -7.0 produces a mass range dispersion of about $0.04 M_{\odot}$ for a given couple of $\log g$ and $T_{\text {eff }}$ values. 
One could speculate if, after deriving a certain mass range for the template white dwarfs assuming a given spread in the $\log q(\mathrm{H})$ values, it is possible to judge if the results and the assumptions about the envelope thickness are consistent with the distribution of the stars in the CMD. As we are going to show now, this is not an easy task when considering realistic errors on the parallax of the objects. We determined, using our cooling tracks, three template sequences of about 100 DA white dwarfs by means of a simplified version of our Monte-Carlo simulator (García-Berro et al. 1999), assuming a negligible error on their colours, and allowing for an error of $\pm 0.10 \mathrm{mag}$ in $M_{V}$, which is approximately the average error of the brightness of the template white dwarfs of Renzini et al. (1996) due to the error on their parallaxes (which is of the order of $5 \%$ ). The first sequence (sequence A) comprises masses in the range between 0.51 and $0.55 M_{\odot}$, and $\log q(\mathrm{H})$ uniformly distributed between -4 and -7 ; sequence $B$ has the same mass range but the value of the thickness of the hydrogen outer layer was kept constant at $\log q(\mathrm{H})=-4$; finally, sequence $\mathrm{C}$ is characterized by $M=0.55 M_{\odot}$ and $\log q(\mathrm{H})=-4$. In Fig. 8 we show the three sequences in the $B V \mathrm{CMD}$ (for $T_{\text {eff }}$ between 10000 and $20000 \mathrm{~K}$ ), after shifting vertically sequence $\mathrm{B}$ by $+0.04 \mathrm{mag}$, and sequence $\mathrm{C}$ by $+0.015 \mathrm{mag}$, in order to reproduce the average brightness of sequence $\mathrm{A}$. The average brightness of the three sequences is different, but the dispersion around the average looks very much the same, and dominated by the parallax error, in spite of the fact that the mass and/or envelope thickness ranges are different.

The main result of this exercise is that uncertainties in the value of $\log q(\mathrm{H})$ produce unavoidable uncertainties on the precise value of the masses of the template white dwarfs and, thus, on the precise location of the template sequence, which may contribute to the error budget of the WD-fitting technique by amounts of the order of less than 0.05 mag. For the sake of conciseness, in the following we will consider as our reference template a sequence (determined from the Monte-Carlo simulations) made of about 100 DA white dwarfs with masses in the range between 0.51 and $0.55 M_{\odot}, \log q(\mathrm{H})$ uniformly distributed between -4 and -7 and effective temperatures in the range between 10000 and $20000 \mathrm{~K}$.

\subsection{Uncertainties on the white dwarf mass and envelope thickness. Clusters with a red $H B$}

We define here as red HB globular clusters those clusters in which the mass of the HB stars is always larger than $\sim 0.52 M_{\odot}$, corresponding to a colour of HB stars larger than $(B-V) \sim-0.21$. This means that all the white dwarfs populating red $\mathrm{HB}$ clusters are a product of AGB evolution (see the discussion in our previous section). In this case, empirical estimates of the white dwarf masses show a range between 0.51 and $0.55 M_{\odot}$. Thus, if the unknown thickness of the $\mathrm{H}$ layers of the DA cluster white dwarfs is similar to that of the local white dwarfs, in prin-

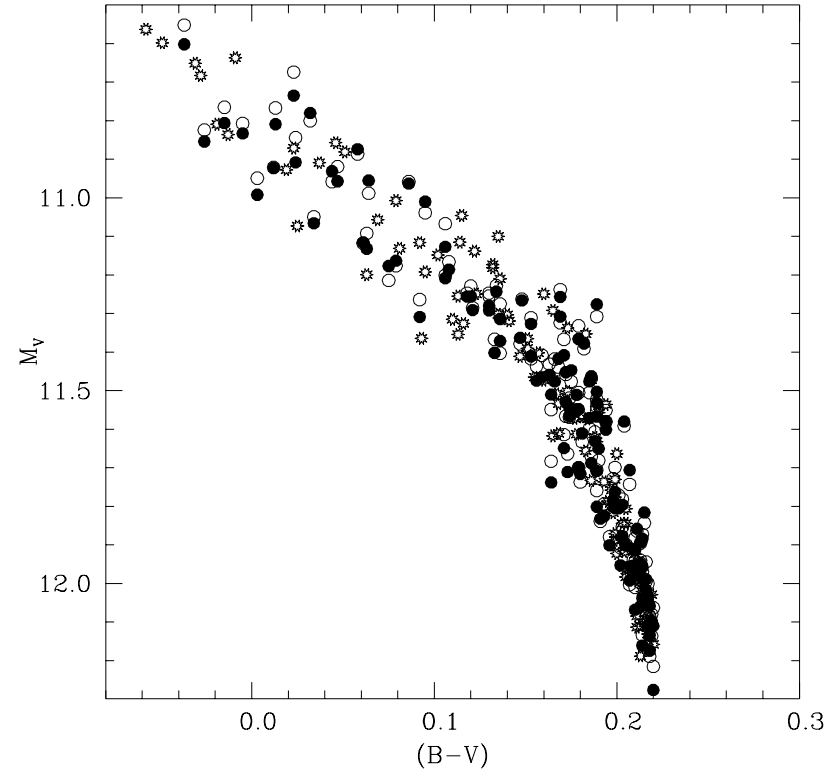

Fig. 8. Comparison among a white dwarf template sequence with masses between 0.51 and $0.55 M_{\odot}$ and $\log q(\mathrm{H})$ between -4 and -7 (sequence $\mathrm{A}$, filled circles), a sequence with the same mass range but $\log q(\mathrm{H})=-4$ (sequence $\mathrm{B}$, empty circles), and a sequence with $M=0.55 M_{\odot}$ and $\log q(\mathrm{H})=-4$ (sequence $\mathrm{C}$, starred circles). All sequences have been calculated including a random error of $0.10 \mathrm{mag}$ in their $M_{V}$ magnitudes. Sequences B and C have been also shifted vertically by, respectively, 0.04 and 0.015 mag in order to overlap with sequence $\mathrm{A}$

ciple there should be no systematic error in the distances derived using our reference template sequence.

To obtain a reasonable estimate of the maximum possible systematic error, our reference sequence has been fitted to a cluster DA sequence with, respectively, $M=0.51 M_{\odot}$ and $\log q(\mathrm{H})=-4$, and $M=0.55 M_{\odot}$ and $\log q(\mathrm{H})=$ -7 . The systematic error in the distance modulus is of $-0.10 \mathrm{mag}$ in the first case and of $+0.10 \mathrm{mag}$ in the second case, with a $\sim 60 \%$ contribution due to the effect of the envelope thickness. It is obvious that in the case that the cluster white dwarfs have an intrinsic spread in mass and/or $\log q(\mathrm{H})$ within the mentioned range, the systematic error on their distance modulus is smaller than $\pm 0.10 \mathrm{mag}$.

If one accepts the results of Alves et al. (2000) about M 15 as due to a genuine spread in the initial-final mass relationship, the mass range of globular cluster white dwarfs could possibly extend up to $0.60 M_{\odot}$. By repeating the previous exercise considering as an extreme case $M=0.60 M_{\odot}$ and $\log q(\mathrm{H})=-7$ for the cluster white dwarfs, one gets systematic errors of $+0.20 \mathrm{mag}$ in the cluster distance modulus. Of course this is a very extreme case. Probably, it is more realistic to consider a mass distribution between 0.51 and $0.60 M_{\odot}$ and $\log q(\mathrm{H})$ distributed between -4 and -7 . With this sample of 100 cluster white dwarfs and using the same reference template white dwarf sequence, we determine a systematic error on the distance modulus of +0.05 mag. Should all the 
globular cluster white dwarfs have the same $\mathrm{H}$ layers thickness, the systematic errors would be then $+0.01 \mathrm{mag}$ if $\log q(\mathrm{H})=-4$ and +0.11 mag if $\log q(\mathrm{H})=-7$.

\subsection{Uncertainties on the white dwarf mass and envelope thickness. Clusters with a blue HB}

We define as blue HB globular clusters those clusters in which the mass of the HB stars extends below $\sim 0.52 M_{\odot}$. In general, even globular clusters with long blue tails always have a fraction of their $\mathrm{HB}$ population located at colours larger than $(B-V) \sim-0.21$, and, therefore, white dwarfs populating clusters with a blue $\mathrm{HB}$ are the product of both AGB evolution and AGB-manqué or post-Early AGB stars. As discussed before, one expects that this kind of globular clusters is populated by white dwarfs with masses down to $\sim 0.45 M_{\odot}$. It appears that blue HB clusters should therefore show a larger mass spread for the white dwarf population, with white dwarf masses ranging between approximately 0.45 and $0.55 M_{\odot}$.

By fitting our template sequence to a cluster sequence with a mass range between 0.45 and $0.55 M_{\odot}$ and a range of $\log q(\mathrm{H})$ as in the template one, we obtained a systematic error on the distance modulus of -0.06 mag. If we accept a larger mass range of up to $0.60 M_{\odot}$, the systematic error goes down to $-0.04 \mathrm{mag}$, since the higher mass objects tend to compensate for the presence of masses lower than the template ones. This is of course just an estimate of the average systematic error in case of globular clusters with a blue HB. To obtain more precise evaluations one should compute synthetic CMDs for each given cluster in order to determine, from the observed HB morphology, the mass distribution along the HB and therefore, on the base of the discussion in Sect. 3.1, infer the possible distribution of white dwarf masses.

Considering now the possibility of different envelope thicknesses between template and cluster white dwarfs, if the white dwarf mass range is between 0.45 and $0.55 M_{\odot}$ and $\log q(\mathrm{H})=-4$ in the cluster, the systematic error on the derived distance modulus is of about $-0.12 \mathrm{mag}$. If we adopt the same mass distribution but we change $\log q(\mathrm{H})$ to -7 , the error is negligible, since the different envelope thickness compensates for the different mass range between the template and cluster sequence.

\subsection{Contamination of the $D A$ sample by non-DA white dwarfs}

Up to know, we have made the assumption that cluster DA white dwarfs can be distinguished from non-DA ones. In absence of spectroscopical identification, the only way to discriminate between DA and non-DA white dwarfs is by checking the relative CMD location of the white dwarf sample (see Fig. 4). Here we estimate the maximum photometric error allowing for a clear distinction between DA and non-DA objects in the $B V$ and VI CMDs. Moreover, we estimate the systematic errors introduced in
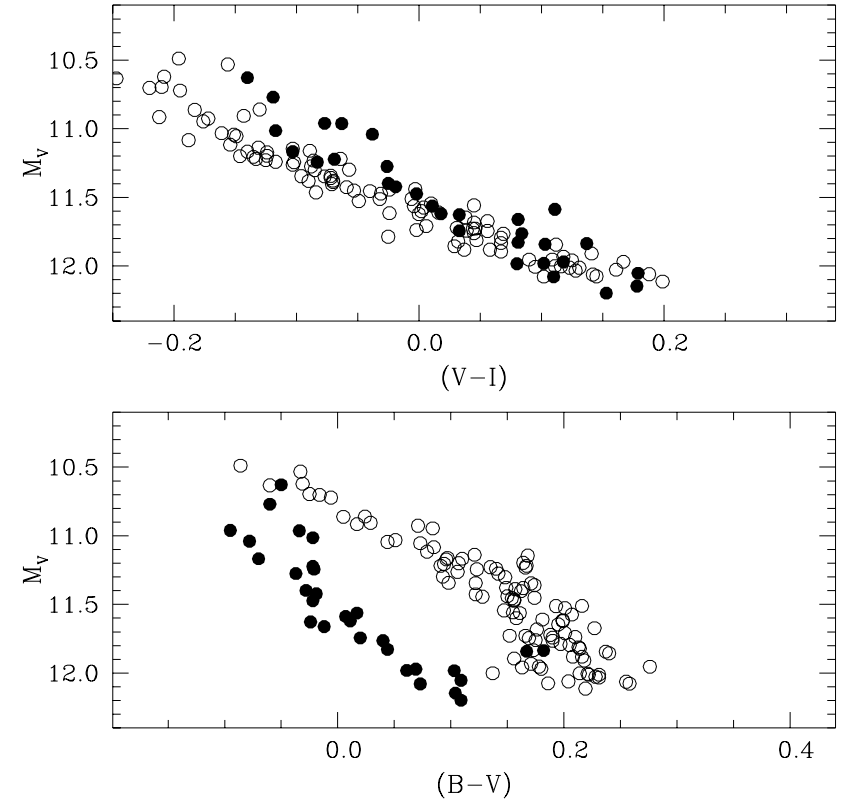

Fig. 9. $B V$ and $V I$ colour-magnitude diagrams for DA (empty circles) and non-DA (filled circles) white dwarfs (see text for details) assuming observational errors of $0.02 \mathrm{mag}$ in $B, V$ and $I$

the globular cluster distances when using a DA template sequence fitted to a cluster white dwarf sample made of both DA and non-DA stars. We assume that the cluster DA/non-DA number ratio in the $T_{\text {eff }}$ interval we are dealing with is $4: 1$ as in the field. Notice, however, that below $T_{\text {eff }}=10000 \mathrm{~K}$ the number of non-DAs is similar or even larger than the number of DAs. The most reasonable explanation for this is that the outer convective region mixes the $\mathrm{H}$ layer into the He envelope (Bergeron et al. 2000).

Figures 9 and 10 show a sequence of about 80 DA and 20 non-DA white dwarfs $\left(M=0.54 M_{\odot}, \log q(\mathrm{H})=\right.$ -4 for the DAs and $\log q(\mathrm{He})=-3.5$ for the non-DAs) computed including $1 \sigma$ photometric errors in $B, V$, and $I$ of, respectively, 0.02 and $0.05 \mathrm{mag}$. Even in the case of the smallest errors, the non-DA and DA sequences are not well separated in the $V I$ plane, while the DA sequence is clearly distinguishable from the non-DA one in the $B V$ plane. With an error bar of the order of 0.05 mag, non-DA white dwarfs start to be mixed up with DA ones also in the $B V$ plane. In order to be more precise, when fitting a template DA sequence to a cluster white dwarf sequence including both DA and non-DA objects, one underestimates the globular cluster distance by only $0.03 \mathrm{mag}$ if the fitting is performed in the $V I$ plane, whereas in the case in which the fitting is performed in the $B V$ plane the distance is overestimated by $\sim 0.20 \mathrm{mag}$. These figures are of course reduced if the ratio DA/non-DA is smaller than the value we have adopted here.

\subsection{He-core white dwarfs}

Another potential source of systematic errors in the WDfitting distances is the presence of $\mathrm{He}^{-}$-core white dwarfs 

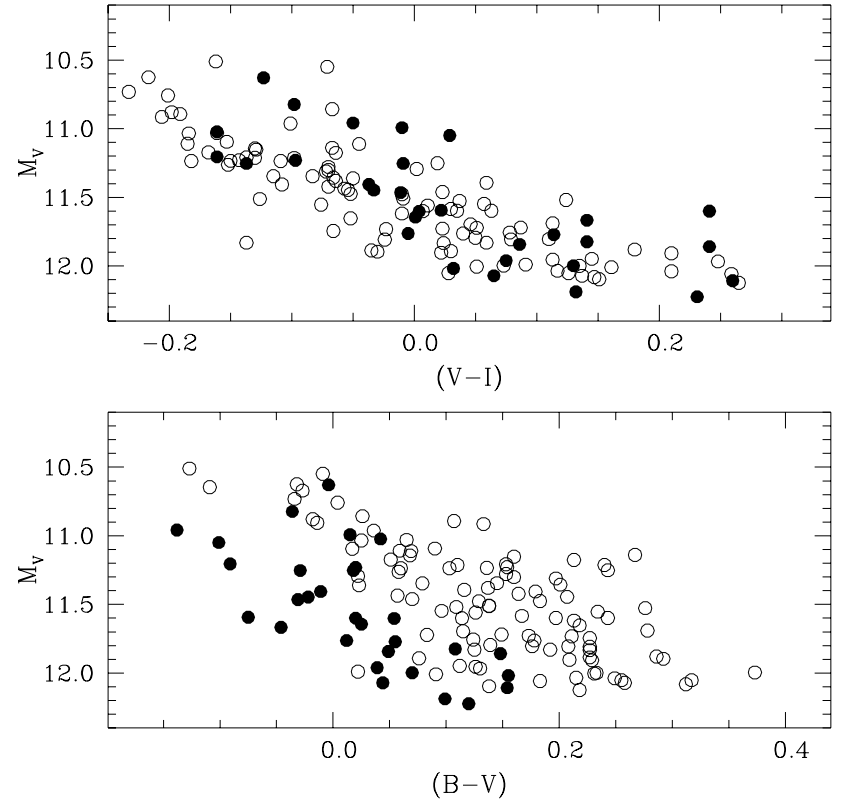

Fig. 10. Same as in Fig. 9 but for observational errors of $0.05 \mathrm{mag}$ in $B, V$ and $I$

(see Fig. 7), and although it is hard to give a precise quantitative assessment of this error source, we can however provide an argument to give some hints about the magnitude of this effect. We will show that the effect would be possibly small or even negligible. White dwarfs with cores made of He are the product of binary evolution. Actual estimates of the binary frequency in globular clusters provide a value of the order of $10 \%$ or less (Hut et al. 1992). If one accepts this estimate, we have verified that, by computing a cluster cooling sequence containing about $130 \mathrm{CO}-$ core white dwarfs with DA/non-DA ratio 4:1 and constant envelope thickness $(\log q(\mathrm{H})=-4$ for the DAs and $\log q(\mathrm{He})=-3.5$ for the non-DAs), plus about 14 (about $10 \%$ of the total sample) He-core DA objects, even if all the $\mathrm{He}-$ core white dwarfs have masses of the order of $0.45 M_{\odot}$, their presence causes a systematic error of the order of only $-0.01 \mathrm{mag}$. In the case that there is a spectrum of masses for $\mathrm{He}$-core white dwarfs, ranging between $\sim 0.20$ and $0.45 M_{\odot}$, then even less objects will be close enough to the CO cooling sequences for the relevant effective temperature range to be confused with them, while the others will lay at larger colours, clearly separated from the more populated CO sequences (see Fig. 11).

\subsection{Cluster reddening}

The knowledge of the reddening of the globular cluster is fundamental in order to derive the cluster distance using the WD-fitting technique. We tested the sensitivity of the derived distances to the uncertainty on the cluster reddening, by considering two DA cooling tracks with $M=$ $0.54 M_{\odot}$ and $\log q(\mathrm{H})=-4$, shifted in colour one with respect to the other; we then fitted the two sequences one of top of the other for different amounts of the relative shift,

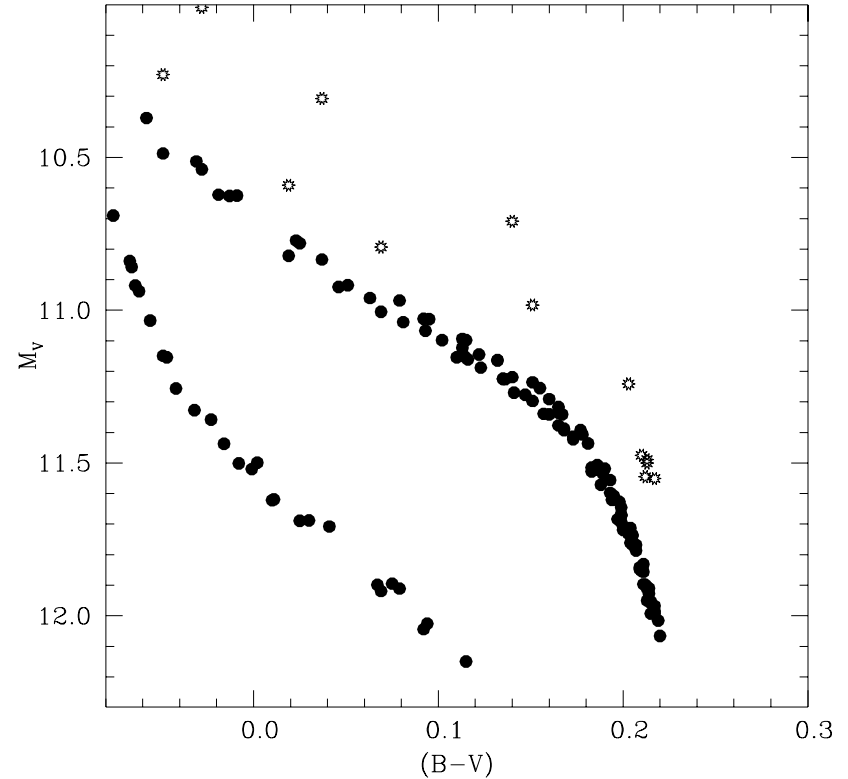

Fig. 11. CMD of a globular cluster cooling sequence made of CO-core white dwarfs with masses between 0.51 and $0.55 M_{\odot}$, DA/non-DA ratio 4:1 (filled circles), plus a $10 \%$ fraction of He-core DA white dwarfs with masses uniformly distributed between 0.20 and $0.45 M_{\odot}$ (starred circles). For all objects the envelope thickness is constant and equal to $\log q(\mathrm{H})=-4$ for the DAs and $\log q(\mathrm{He})=-3.5$ for the non-DAs

obtaining in the $B V$ and $V I$ planes a value for the derivative of the apparent distance modulus with respect to the $(B-V)$ colour excess, $\Delta(m-M)_{V} / \Delta E(B-V) \sim-5.5$.

\section{Summary and conclusions}

In this paper we have thoroughly discussed many possible sources of systematic errors on the globular clusters distances obtained using the WD-fitting technique. In order to do so we have first investigated in detail the changes produced in the $B V$ and $V I$ CMDs when varying the adopted mass of the template white dwarf cooling sequence, the influence of the assumed thicknesses of their envelopes on the location of the cooling tracks, as well as the consequences of changing the chemical composition of their envelopes and of their cores. We have then applied these results in order to derive, by means of Monte-Carlo simulations, a realistic estimate of the systematic errors involved in the application of this technique to galactic globular clusters. Our main results can be summarized as follows:

1. The unknown thickness of the H layers in cluster DA white dwarfs plays a non-negligible role, comparable to the role played by uncertainties on the white dwarf masses. For reasonable assumptions (derived from observations of field white dwarfs and constraints from globular cluster CMDs) about the unknown mass and $\log q(\mathrm{H})$ values in cluster DA white dwarfs, a realistic estimate of the maximum systematic error on the derived distance moduli is within \pm 0.10 mag. However, 
one should be aware that particular combinations of white dwarf masses and envelope thicknesses - still allowed by current weak or non-existent observational constraints - could produce larger errors.

2. A photometric precision better than $\sim 0.05 \mathrm{mag}$ is needed in order to distinguish DA from non-DA white dwarfs in the $B V$ plane. An even better precision is needed when using the $V I$ plane. In the case of larger observational errors and no clear (spectroscopical) distinction between DA and non-DA cluster white dwarfs, fitting a template DA sequence to a cluster sequence made of a mixture of DA and non-DA stars introduces a very small systematic error $\sim-0.03 \mathrm{mag}$ in the $V I$ plane, but this error amounts to $\sim+0.20 \mathrm{mag}$, in the $B V$ plane. The $V I$ plane looks therefore better suited for the application of the WD-fitting method, since it permits to get rid of the uncertainty due to a possible contamination of the DA sample by non-DA white dwarfs.

3. Contamination by $\mathrm{He}-$ core white dwarfs should not influence appreciably the WD-fitting distances.

4. Due to the steep slopes of the white dwarf cooling curves in the CMD, the distance derived from the WDfitting technique has a non negligible dependence on the adopted cluster reddening. We obtained a derivative $\Delta(m-M)_{V} / \Delta E(B-V) \sim-5.5$, a dependence which is similar to the one of the MS-fitting technique in the $B V \mathrm{CMD}$.

Acknowledgements. We thank M. Zoccali for useful comments to a preliminary version of the manuscript. Part of this work was supported by the Spanish DGES project numbers PB981183-C03-02, AYA2000-1785 and ESP98-1348, by the CIRIT and by Sun MicroSystems under the Academic Equipment Grant AEG-7824-990325-SP. One of us (S.C.) has been supported by MURST-Cofin2000- under the scientific project "Stellar Observables of Cosmological Relevance".

\section{References}

Alves, D. R., Bond, H. E., \& Livio, M. 2000, AJ, in press

Barstow, M. A., Fleming, T. A., Diamond, C. J., et al. 1993, MNRAS, 264, 16

Bergeron, P., Saumon, D., \& Wesemael, F. 1995, ApJ, 443, 764

Bergeron, P., Wesemael, F., \& Beauchamp, A. 1995, PASP, 107, 1047

Bergeron, P., Leggett, S. K., \& Ruiz, M. T. 2000 [astro-ph/0011286]

Bono, G., Caputo, F., Cassisi, S., Castellani, V., \& Marconi, M. 1997, ApJ, 479, 279

Bragaglia, A., Renzini, A., \& Bergeron, P. 1995, ApJ, 443, 735

Cassisi, S., Castellani, V., Degl'Innocenti, S., Salaris, M., \& Weiss, A. 1999, A\&AS, 134, 103

Cassisi, S., Castellani, V., Degl'Innocenti, S., Piotto, G. P., \& Salaris, M. 2001, A\&A, 366, 578

Castellani, V., Degl'Innocenti, S., \& Romaniello, M. 1994a, ApJ, 423, 266

Castellani, V., Luridiana, V., \& Romaniello, M. 1994b, ApJ, 428,633
Clemens, J. C. 1995, in White Dwarfs, ed. D. Koester, \& K. Werner (Springer), 294

D’Antona, F., \& Mazzitelli, I. 1990, ARA\&A, 28, 139

Dorman, B., Rood, R. T., \& O'Connell, R. W. 1993, ApJ, 419, 596

Fernley, J., Barnes, T. G., Skillen, I., et al. 1998, A\&A, 330, 515

García-Berro, E., Torres, S., Isern, J., \& Burkert, A. 1999, MNRAS, 302, 173

Gratton, R. G., Fusi-Pecci, F., Carretta, E., et al. 1997, ApJ, 491, 749

Greggio, L., \& Renzini, A. 1990, ApJ, 364, 527

Hansen, B. M. S. 1999, ApJ, 520, 680

Hansen, B. M. S., \& Phinney, E. S. 1998, MNRAS, 294, 557

Hubbard, W. B., \& Lampe M. 1969, ApJS, 18, 297

Hut, P., McMillan, S., Goodman, J., et al. 1992, PASP, 104, 981

Iglesias, C. A., \& Rogers, F. J. 1993, ApJ, 412, 752

Isern, J., García-Berro, E., Hernanz, M., Mochkovitch, R., \& Torres, S. 1998, ApJ, 486, 413

Itoh, N., Mitake, S., Iyetomi, H., \& Ichimaru, S. 1983 ApJ, 273,774

Itoh, N., Hayashi, H., Nishikawa, A., \& Kohyama, Y. 1996, ApJS, 102, 411

Kippenhahn, R., Kohl, K., \& Weigert, A. 1967, Z. Ap., 66, 58

Koester, D., \& Chanmugam, G. 1990, Rep. Prog. Phys., 53, 837

Luri, X., Gómez, A. E., Torra, J., Figueras, F., \& Mennessier, M. O. 1998, A\&A, 335, L81

MacDonald, J., Hernanz, M., \& Jose, J. 1998, MNRAS, 296, 523

Mazzitelli, I., D'Antona, F., \& Caloi, V. 1995, A\&A, 302, 382

Michaud, G., Bergeron, P., Wesemael, F., \& Fontaine, G. 1985, ApJ, 299, 741

Moehler, S., Heber, U., Napiwotzki, R., Koester, D., \& Renzini, A. 2000, A\&A, 354, L75

Pelletier, C., Fontaine, G., Wesemael, F., Michaud, G., \& Wegner, G. 1986, ApJ, 307, 242

Provencal, J. L., Shipman, H. L., Hog, E., \& Thejll, P. 1998, ApJ, 494, 759

Reid, I. N. 1996, AJ, 111, 2000

Renzini, A., Bragaglia, A. Ferraro, F. R., et al. 1996, ApJ, 465, L23

Richer, H. B., et al. 1997, ApJ, 484, 741

Salaris, M., \& Weiss, A. 1997, A\&A, 327, 107

Salaris, M., \& Weiss, A. 1998, A\&A, 335, 943

Salaris, M., Domínguez, I., García-Berro, E., et al. 1997, ApJ, 486,413

Salaris, M., García-Berro, E., Hernanz, M., Isern, J., \& Saumon, D. 2000, ApJ, 544, 1036

Saumon, D., Chabrier, G., \& Van Horn, H. M. 1995, ApJS, 99, 713

Segretain, L., Chabrier, G., Hernanz, M., et al. 1994, ApJ, 434, 641

Umeda, H., Nomoto, K., Yamaoka, H., \& Wanajo, S. 1999, ApJ, 513, 861

VandenBerg, D. A., Swenson, F. J., Rogers, F. J., Iglesias, C. A., \& Alexander, D. R. 2000, ApJ, 532, 430

von Hippel, T., \& Gilmore, G. 2000, AJ, 120, 1384

Wagenhuber, J. 1996, Ph.D. Thesis, Max-Planck-Institut für Astrophysik 